\title{
Test de marcha de seis minutos en niños con bronquiolitis obliterante postviral. Correlación con espirometría
}

\author{
DANIEL ZENTENO A.*, HOMERO PUPPO G.**, RAMIRO GONZÁLEZ V.***, \\ DOLORES PAVÓN T.***, ROBERTO VERA U.****, RODRIGO TORRES C.****, \\ CHUNG KUO K.****, MARÍA ANGÉLICA PÉREZ H.*** y GUIDO GIRARDI B.***
}

Six minute walk test in children with post-infectious obliterans bronchiolitis. Its relation with spirometry

Post infectious obliterans bronchiolitis $(O B)$ causes persistent pulmonary function impairment and could affect patient ability to perform exercise. Six minute-walk test (6MWT) is a useful tool to study these aspects. Objective: Children with OB were evaluated with spirometry and 6MWT and the relationship between spirometrics measurements and 6MWT were determined. Twenty-seven children with $O B$ were studied with a base line spirometry and 6MWT. Correlation between spirometry and 6MWT: covered distance, Borg index (BI), oxygen saturation $\left(\mathrm{O}_{2} \mathrm{~S}\right)$ and heart rate $(\mathrm{HR})$, were carried out using Rho of Spearman with SPSS 11.5. There was not relation between the covered distance and spirometrics values; only FVC correlates with final $\mathrm{O}_{2} \mathrm{~S}(\mathrm{rho}=0.4 ; p=0.02)$. The other spirometrics variables correlates with $\mathrm{BI}, \mathrm{O}_{2} \mathrm{~S}$ and $\mathrm{HR}$; Interestingly $\mathrm{FEV} \mathrm{V}_{1}$ correlates with: $\mathrm{BI}$ (rho $=-0.7 ; p<0.01), \mathrm{O}_{2} S$ (rho $=0.5 ; p<0.01$ ) and $\mathrm{HR}$ (rho $=-0.5 ; p<0.01$ ). BI was significant related to $F E V_{1} / F V C$ (rho $=-0.7 ; p<0.01$ ) and $F E F_{25-75}$ (rho $=-0.8 ; p<0.01$ ). Airflow obstruction, especifically a low $F E V_{1}$, is related with a high BI and HR; and also with a lower $\mathrm{O}_{2} \mathrm{~S}$ at the end of $6 \mathrm{MWT}$. Increase of $\mathrm{BI}$ is the change most related with airflow obstruction.

Key words: Bronchiolitis obliterans, six-minute walk test, adenovirus.

\section{Resumen}

La Bronquiolitis Obliterante (BO) postviral produce alteraciones en la función pulmonar que pueden repercutir en la capacidad para realizar ejercicio, la cual se puede evaluar con el test de marcha de 6 minutos (TM6). Objetivo: Estudiar con esta prueba a niños con BO postviral y determinar la relación entre variables espirométricas y los resultados del TM6. Veintisiete pacientes, se estudiaron con espirometría basal y TM6. Se relacionaron variables espirométricas con resultados del TM6: distancia caminada, índice de Borg (IB), saturación de oxígeno $\left(\mathrm{SaO}_{2}\right)$ y frecuencia cardíaca (FC), mediante Rho de Spearman utilizando SPSS 11,5. No hubo correlación entre la distancia caminada y ninguna variable espirométrica; la CVF sólo se correlacionó con $\mathrm{SaO}_{2}$ final (rho $=0,4 ; p=0,02)$. El resto de las variables espirométricas se correlacionaron con $\mathrm{IB}, \mathrm{SaO}_{2}$ y FC; destacando $V E F_{1}$ con: IB (rho = -0,7; $\left.p<0,01\right), \mathrm{SaO}_{2}($ rho $=0,5 ; p<0,01)$ y FC (rho = -0,5; $p<0,01)$. El IB también se correlacionó significativamente con $V E F_{1} / C V F$ (rho $\left.=-0,7 ; p<0,01\right)$ y $\mathrm{FEF}_{25-75}$ (rho $\left.=-0,8 ; p<0,01\right)$. La obstrucción al flujo aéreo, en especial un menor $V E F_{1}$, se relaciona con altos IB y FC; además con una menor $\mathrm{SaO}_{2}$ al final del TM6. El aumento del IB es la variable que más se relacionó con obstrucción al flujo aéreo.

Palabras clave: Bronquiolitis obliterante, test de marcha seis minutos, adenovirus.

Unidad de Broncopulmonar, Hospital Exequiel González Cortés, Santiago, Chile. Departamento de Pediatría Sur, Facultad de Medicina, Universidad de Chile.

* Pediatra. Becado de Neumología, Departamento de Pediatría Sur, Facultad de Medicina, Universidad de Chile. Unidad de Broncopulmonar, Hospital Exequiel González Cortés, Santiago, Chile.

** Kinesiólogo, Facultad de Medicina, Universidad de Chile, Unidad de Kinesiología, Hospital Exequiel González Cortés, Santiago, Chile.

*** Pediatra Broncopulmonar, Departamento de Pediatría Sur, Facultad de Medicina, Universidad de Chile. Unidad de Broncopulmonar, Hospital Exequiel González Cortés, Santiago, Chile.

**** Kinesiólogo, Programa Nacional de Ventilación Mecánica No-Invasiva, Santiago, Chile. 


\section{Introducción}

$\mathrm{El}$ adenovirus (ADV) es causante del 3 al 5\% de las infecciones respiratorias agudas bajas que requieren hospitalización en Chile. La neumonía por ADV suele ser grave, puede llegar al distrés y acompañarse de compromiso multisistémico. La mortalidad es de 10 a 15\%, y puede dejar secuelas importantes hasta en el 50\% de los casos, donde destacan: bronquiolitis obliterante (BO), bronquiectasias, pulmón hiperlúcido y dependencia de oxígeno. La BO es la segunda causa más importante de enfermedad pulmonar crónica en nuestro país y una entidad frecuente en el cono sur de América ${ }^{1-3}$.

Las alteraciones espirométricas en niños con BO presentan un patrón de alteración ventilatoria obstructiva difusa, en los casos severos con capacidad vital forzada (CVF) disminuida. La respuesta a broncodilatadores varía de nula a significativa, pero sin normalizar valores ${ }^{1,4}$. Muchos pacientes con compromiso importante de la función pulmonar no relatan dificultades con el ejercicio, por lo cual está indicado hacer una medición objetiva como el test de marcha.

El test de marcha de seis minutos (TM6) es un test submáximo, diseñado originalmente para adultos; que evalúa la capacidad para realizar ejercicio, la cual a su vez se relaciona con la calidad de vida. Su bajo costo, fácil realización, alta reproducibilidad y buena correlación con actividades de la vida diaria, lo sitúa como el test de caminata ideal ${ }^{5,6}$.

Está validado como indicador de capacidad física en múltiples patologías y a distintas edades. En adultos está indicado en comparaciones pre y postratamiento (ej. trasplante pulmonar, cirugía de reducción de volumen, rehabilitación pulmonar), valoración del estado funcional (ej. EPOC, fibrosis quística, hipertensión pulmonar, insuficiencia cardíaca) y como predictor de morbilidad y mortalidad (ej. EPOC, hipertensión pulmonar, insuficiencia cardíaca $)^{7,8}$.

Ha sido validado para niños sanos en estudios internacionales ${ }^{6}$ y para niños sanos chilenos ${ }^{9}$. Pese a esto, su utilización en Pediatría aún es limitada y los estudios son escasos. Es indicado principalmente en niños con fibrosis quística ${ }^{10}$, pero también ha demostrado su utilidad en niños cardiópatas $^{11}$ y niños pretrasplante cardíaco y/o pulmonar ${ }^{12}$.

No existen datos sobre las alteraciones en el TM6 en niños con BO postviral. Tampoco se conoce la correlación que pueda existir entre la capacidad física, evaluable con el TM6, y las variables espirométricas.
El objetivo de este estudio fue evaluar con el TM6 a niños con BO postviral y determinar la relación entre variables espirométricas y los resultados del TM6.

\section{Pacientes y Métodos}

Estudio de cohorte longitudinal, donde se estudiaron 27 niños con BO postviral. Se incluyeron niños de 8 a 14 años, con antecedentes de neumonía severa por ADV durante sus primeros años de vida, con cuadro clínico y Tomografía Axial Computada (TAC) compatible con BO; controlados periódicamente en la Unidad de Broncopulmonar, Hospital Exequiel González Cortés, Santiago, Chile.

Se excluyeron pacientes con inestabilidad o reagudización de su patología respiratoria, con otras causas de daño pulmonar crónico (ej: fibrosis quística, disquinesia ciliar, displasia broncopulmonar) y con otras patologías crónicas que pudiesen influir en la capacidad física (ej: enfermedades neuromusculares, cardiopatías).

Se realizó espirometría basal y dentro de un intervalo menor de 1 semana, se practicó TM6; según normas técnicas de la ATS $^{13,8}$. Para su análisis se utilizaron los valores de referencia de Gutiérrez $^{14}$ en la espirometría y de Escobar ${ }^{9}$ para TM6.

Luego se relacionaron los valores porcentuales del predicho de $\mathrm{VEF}_{1}, \mathrm{CVF}, \mathrm{VEF}_{1} / \mathrm{CVF}$ y $\mathrm{FEF}_{25-75 \%}$ con la diferencia porcentual entre la distancia esperada y caminada (DC), índice de Borg (IB), saturación de oxígeno $\left(\mathrm{SaO}_{2}\right)$ y frecuencia cardíaca (FC) al terminar el TM6; mediante el coeficiente de correlación Rho de Spearman, considerando significativos $\mathrm{p}<0,05$. Para este análisis se utilizó el programa SPSS 11,5 para Windows.

\section{Resultados}

La edad promedio de los niños fue $12 \pm 2$ años (rango 8 a 14), 18 varones. Tres niños utilizaban oxígenoterapia domiciliaria en el momento del estudio (Tabla 1).

En la espirometría se encontró alteración ventilatoria obstructiva en todos los pacientes. Esta fue, de grado avanzado en 11 niños, moderado en 5, leve en 8 y mínimo en 3. La CVF estuvo disminuida en 11 de ellos, principalmente pertenecientes al grupo con obstrucción al flujo aéreo de grado avanzado. La Tabla 2 muestra los valores promedios de $\mathrm{CVF}, \mathrm{VEF}_{1}, \mathrm{VEF}_{1} / \mathrm{CVF}$ y $\mathrm{FEF}_{25-75 \% \text {. }}$ 
Tabla 1. Características de los 27 niños con bronquiolitis obliterante postviral

\begin{tabular}{ll}
\hline $\begin{array}{l}\text { Edad promedio } \\
\text { Género }\end{array}$ & $12 \pm 2$ años \\
$\quad$ Femenino & $9(33 \%)$ \\
$\quad$ Masculino & $18(67 \%)$ \\
Talla & $143 \pm 13 \mathrm{~cm}$ \\
Peso & $40,3 \pm 11 \mathrm{~kg}$ \\
Uso de oxígeno domiciliario & $3(11 \%)$ \\
\hline
\end{tabular}

Variable de dispersión: desviación estándar.

Tabla 3. Resultados de los TM6 de los 27 niños con bronquiolitis obliterante postviral

\begin{tabular}{lcr}
\hline Índice & Inicial & \multicolumn{1}{c}{ Final } \\
\hline $\mathrm{SaO}_{2}(\%)$ & $97 \pm 1$ & $95 \pm 4$ \\
$\mathrm{IB}$ & $0,9 \pm 1$ & $6,0 \pm 2$ \\
$\mathrm{FC}(\mathrm{lpm})$ & $91 \pm 12$ & $138 \pm 20$ \\
Distancia caminada (m) & - & $598 \pm 71$ \\
\hline
\end{tabular}

$\mathrm{SaO}_{2}$ : Saturación de oxígeno. IB: índice de Borg. FC: Frecuencia cardíaca. Variable dispersión: desviación estándar.

Los resultados del TM6 demostraron que la distancia caminada promedio fue $598 \pm 71$ metros. Al considerar que el límite inferior esperado promedio era $690 \pm 43$ metros, se establece que la distancia recorrida promedio era un $13 \%$ menor al límite inferior esperado. Los niños con alteración ventilatoria obstructiva avanzada y CVF disminuida mostraron una tendencia a caminar una distancia menor.
Tabla 2. Alteraciones espirométricas de los 27 niños con BO postviral

\begin{tabular}{lc}
\hline Índice & Promedios (\%) \\
\hline $\mathrm{CVF}$ & $85,4 \pm 15$ \\
$\mathrm{VEF}_{1}$ & $58,3 \pm 23$ \\
$\mathrm{VEF}_{1} / \mathrm{CVF}$ & $59,3 \pm 32$ \\
$\mathrm{FEF}_{25-75}$ & $31,7 \pm 25$ \\
\hline
\end{tabular}

CVF: capacidad vital forzada. $\mathrm{VEF}_{1}$ : volumen espiratorio forzado del primer segundo. $\mathrm{VEF}_{1} / \mathrm{CVF}$ : relación entre $\mathrm{VEF}_{1}$ y CVF. $\mathrm{FEF}_{25-75 \%}$ : flujo espiratorio forzado al 25$75 \%$ de la CVF. Variable de dispersión: desviación estándar.

El resto de los parámetros evaluados en el TM6: $\mathrm{SaO}_{2}$, índice de Borg, frecuencia cardíaca fueron registrados al inicio y final del test; sus resultados se muestran en la Tabla 3.

Tres niños disminuyeron su $\mathrm{SaO}_{2}$ a $<90 \%$, post TM6. Estos terminaron sin problemas el test y fueron manejados con medidas generales y apoyo con oxígenoterapia, por algunos minutos, hasta que recuperaron su condición basal. Solo uno de ellos pertenecía al grupo de los oxígeno dependientes.

La Tabla 4 muestra las correlaciones entre las variables espirométricas $\left(\mathrm{VEF}_{1}, \mathrm{VEF}_{1} / \mathrm{CVF}\right.$, $\mathrm{FEF}_{25-75 \%}$ ) y los resultados finales del TM6 (DC, $\mathrm{IB}, \mathrm{SaO}_{2}$ y FC) con sus valores de significancia estadística correspondientes.

No existió correlación entre DC y ninguna variable espirométrica. La CVF sólo se correlacionó con la $\mathrm{SaO}_{2}$ final. El resto de las variables espirométricas $\left(\mathrm{VEF}_{1}, \mathrm{VEF}_{1} / \mathrm{CVF}, \mathrm{FEF}_{25-75}\right)$ se correlacionaron con el IB, $\mathrm{SaO}_{2}$ y FC al finalizar el TM6; destacando correlaciones altamente significativas del $\mathrm{VEF}_{1}$ con el $\mathrm{IB}, \mathrm{SaO}_{2}$ y FC, ade-

Tabla 4. Correlaciones (rho de Spearman) entre variables espirométricas y variables finales del TM6 de los 27 niños con bronquiolitis obliterante postviral

\begin{tabular}{lllcl}
\hline & $\boldsymbol{\Delta C}$ & IB final & $\mathbf{S a O}_{2}$ final & FC final \\
\hline $\mathrm{CVF}$ & 0,05 & $-0,4$ & $0,4^{*}$ & $-0,3$ \\
$\mathrm{VEF}_{1}$ & 0,2 & $-0,7^{* *}$ & $0,5^{* *}$ & $-0,5^{* *}$ \\
$\mathrm{VEF}_{1} / \mathrm{CVF}$ & 0,3 & $-0,7^{* *}$ & $0,4^{*}$ & $-0,4^{*}$ \\
$\mathrm{FEF}_{25-75 \%}$ & 0,3 & $-0,8^{* *}$ & $0,4^{*}$ & $-0,5^{*}$ \\
\hline
\end{tabular}

CVF: capacidad vital forzada. $\mathrm{VEF}_{1}$ : Volumen espiratorio forzado del primer segundo. $\mathrm{VEF}_{1} / \mathrm{CVF}$ : Relación entre $\mathrm{VEF}_{1}$ y CVF. $\mathrm{FEF}_{25-75 \%}$ : Flujo espiratorio forzado al 25-75\% de la CVF. $\triangle \mathrm{C}$ : diferencia porcentual entre la distancia esperada y caminada. IB: Índice de Borg. $\mathrm{SaO}_{2}$ : Saturación de oxígeno. FC: Frecuencia cardíaca. *p < 0,05; $* *$ p $<0,01$. 
más del IB con todas las variables espirométricas (excepto CVF).

\section{Discusión}

Nuestro estudio fue realizado en una población homogénea de niños con BO postviral, específicamente por ADV. Todos presentaban alteraciones espirométricas similares a las encontradas en otros estudios ${ }^{1,4}$; es decir, con un patrón obstructivo de grado variable con o sin disminución de la CVF. El 40\% presentaba un grado avanzado de obstrucción y el 37\% CVF disminuida.

El TM6 en los niños con BO, fue bien tolerado, no se presentaron complicaciones; situación similar a la encontrada en niños con enfermedad grave (pretrasplante cardíaco y/o pulmonar), cardiopatías y fibrosis quística ${ }^{10-12}$. Resultó además de fácil realización y bajo costo. Estas características sumadas a la alta relación con las actividades de la vida cotidiana, sitúan al TM6 como el test de caminata ideal según revisiones recientes $^{5}$ y nos llevan a considerarlo de uso rutinario en las patologías mencionadas en niños, como método para evaluar su capacidad funcional, abriéndonos además la posibilidad de estudio en otras patologías crónicas donde se ha demostrado su utilidad en adultos como el caso de la fibrosis pulmonar idiopática, situación en que recientemente se demostró que el TM6 puede ser utilizado como predictor independiente de mortalidad ${ }^{15}$.

Si bien la distancia caminada fue un $13 \%$ menor a la distancia esperada considerando para esta diferencia el límite inferior esperado; no se encontró correlación con ninguna de las variables espirométricas, situación similar a la encontrada en varios estudios realizados en adultos con EPOC ${ }^{16-18}$; sin embargo, otros estudios han encontrado relación con la CVF, $V_{E F}$ y difusión de $\mathrm{CO}^{19,20}$. Esta última es la que más constantemente se asocia a la distancia caminada; la cual, no fue evaluada en nuestros pacientes.

Con respecto a la relación de las variables espirométricas relacionadas con obstrucción al flujo aéreo $\left(\mathrm{VEF}_{1}, \mathrm{VEF}_{1} / \mathrm{CVF}, \mathrm{FEF}_{25-75 \%}\right)$, se encontraron correlacionadas con un IB y FC mayores, además de una menor $\mathrm{SaO}_{2}$ al final del TM6. Destacaba especialmente la relación del $\mathrm{VEF}_{1}$ con estas variables. El IB como parámetro final del TM6 fue el que se encontró más relacionado con obstrucción al flujo aéreo $\left(\mathrm{VEF}_{1}\right.$, $\left.\mathrm{VEF}_{1} / \mathrm{CVF}, \mathrm{FEF}_{25-75}\right)$. La CVF sólo se correlacionó con la $\mathrm{SaO}_{2}$ al final del test.
Dentro de las limitaciones del estudio mencionamos el número reducido de sujetos $(\mathrm{n}=27)$, explicable por los criterios de inclusión y exclusión utilizados y por el rango de edad para el cual está validado el TM6 en niños chilenos.

Este trabajo es un aporte a la literatura, ya que no conocemos estudios similares en niños con BO. Este sería el primer estudio que relaciona la función pulmonar con la capacidad para realizar ejercicios en niños con BO postviral.

Nuestro estudio aporta una herramienta adicional a la evaluación clásica de la función pulmonar, que permite tener una visión más completa de la capacidad funcional de estos pacientes y estimar mejor su desempeño en las actividades cotidianas.

En conclusión, el TM6 en niños con BO es de bajo costo, bien tolerado, se puede realizar sin complicaciones y permite detectar las limitaciones para realizar ejercicio. Al evaluar una espirometría de niños con BO postviral, el análisis del $\mathrm{VEF}_{1}$ es el parámetro que más se relaciona con su capacidad funcional. A menor $\mathrm{VEF}_{1}$ : menor $\mathrm{SaO}_{2}$, mayor disnea y mayor FC; no se relaciona con la distancia caminada. Un mayor grado de disnea se relaciona en forma importante con la obstrucción al flujo aéreo.

\section{Agradecimientos}

Agradecemos al Prof. Dr. Arnoldo Quezada L. (Pediatra Inmunólogo-Neumólogo, Universidad de Chile, Hospital Exequiel González Cortés, Santiago) por la revisión del trabajo; a los médicos, kinesiólogos y personal de nuestra Unidad de Broncopulmonar, Laboratorio de Función Pulmonar y Unidad de Kinesiología que día a día atienden a estos niños. Finalmente a nuestros pacientes y sus padres, que gracias a su colaboración permitieron concretar este trabajo.

\section{Bibliografía}

1.- CASTRO-RODRÍGUEZ J A, DASZENIES C, GARCÍA M, MEYER R, GONZÁLEZ R. Adenovirus Pneumonia in Infants and Factors for Developing Bronchiolitis Obliterans: A 5-Year Follow-Up. Pediatr Pulmonol 2006; 41: 947-53.

2.- PALOMINO M A, LARRAÑAGA C, VILLAGRA E, CAMACHO J, AVENDAÑO L F. Adenovirus and respiratory syncytial virus-adenovirus mixed acute lower respiratory infections in Chilean infants. Pediatr Infect Dis J 2004; 23: 337-41.

3.- LARRAÑAGA C, KAJON A, VILLAGRA E, AVENDAÑO L F. Adenovirus surveillance on children hospitalized for acute lower respiratory infections in 
Chile (1988-1996). J Med Virol 2000; 60: 342-6.

4.- JONES M H, PITREZ P M, STEIN R T. Post-infectious bronchiolitis obliterans. Pediatr Pulmonol 2004; 26 Suppl 1: 64-5.

5.- SOLWAY S, BROOKS D, LACASSE Y, THOMAS S. A qualitative systemic overview of the measurement properties of functional walk tests used in the cardiorespiratory domain. Chest 2001; 119: 256-70.

6.- $\quad$ LI A M, YIN J, YU C C, TSANG T, SO H K, WONG E, et al. The six-minute walk test in healthy children: reliability and validity. Eur Respir J 2005; 25: 105760.

7.- CARTER R, HOLIDAY D, NWASURUBA C, STOCKS J, GROTHUES C, TIEP B. 6-Minute Walk Work for Assessment of Functional Capacity in Patients With COPD. Chest 2003; 123: 1408-15.

8.- ATS statement: guidelines for the six-minute walk test. Am J Respir Crit Care Med 2002; 166: 111-7.

9.- ESCOBAR M, LÓPEZ A, VELIZ C, CRISTOMO S, PINOCHET R. Test de Marcha en 6 minutos en niños Chilenos. Kinesiología, 2001; 62: 16-20.

10.- GULMANS V A, VAN VELDHOVEN N H, DE MEER K, HELDERS P J. The six-minute walking test in children with cystic fibrosis: reliability and validity. Pediatr Pulmonol 1996; 22: 85-9.

11.- MOALLA W, GAUTHIER R, MAINGOURD Y, AHMAIDI S. Six-Minute Walking Test to Assess Exercise Tolerance and Cardiorespiratory Responses During Training Program in Children With Congenital Heart Disease. Int J Sports Med 2005; 26: 756-62.

12.- NIXON P A, JOSWIAK M L, FRICKER F J. A sixminute walk test for assessing exercise tolerance in severely ill children. J Pediatr 1996; 129: 362-6.

13.- MILLER M R, HANKINSON J, BRUSASCO V, BURGOS F, CASABURI R, COATES A. Standardisation of spirometry. Eur Respir J 2005; 26: 319-38.

14.- GUTIÉRREZ M, RIOSECO F, ROJAS A, CASANOVAD. Determinación de valores espirométricos en una población chilena normal mayor de 5 años, a nivel del mar. Rev Méd Chile 1996; 124: 1295-306.

15.- LEDERER D J, ARCASOY S M, WILT J S, D'OVIDIO F, SONETT J R, KAWUT S M. Six-Minute-Walk Distance Predicts Waiting List Survival in Idiopathic Pulmonary Fibrosis. Am J Respir Crit Care Med 2006; 174: 65964.

16.- TEIXEIRA P J, COSTA C C, BERTON D C, VERSA G, BERTOLETTI O, CANTERLE D B. Six-minute walk work is not correlated to the degree of airflow obstruction in patients with Chronic Obstructive Pulmonary Disease. Rev Port Pneumol 2006; 12: 241-54.

17.- KILLIAN K H, CAMPBELL E J M, SUMMERS E, JONES N L. Relationship between pulmonary impairment, exercise capacity and intensity of dyspnea. Eur Respir J 1993; 6 (Suppl 7): 571S.

18.- FINK G, MOSHE S, GOSHEN J, KLAINMAN E, LEBZELTER J, SPITZER S, et al. Functional Evaluation in Patients with Chronic Obstructive Pulmonary Disease: Pulmonary Function Test versus Cardiopulmonary Exercise Test. J Occup Environ Med 2002; 44: 54-8.

19.- MAC GAVIN C R, ARTVINLI M, NAOE H, MCHARDY G J. Dyspnoea, disability, and distance walked: comparison of estimates of exercise performance in respiratory disease. Br Med J 1978; 2: 2413.

20.- MAK V H, BUGLER J R, ROBERTS C M, SPIRO S G. Effect of arterial oxygen desaturation on six minute walk distance, perceived effort, and perceived breathlessness in patients with airflow limitation. Thorax 1993; 48: 33-8.

Correspondencia a:

Dr. Daniel Zenteno A.

Román Díaz 175, Dpto. 706, Providencia,

Santiago, Chile

Fax: 00-56-02-4605381 / 00-56-02-2298665

E-mail: danielzenteno@gmail.com 